\title{
LA MORFOLOGÍA DE LAS LENGUAS ARTIFICIALES. EL CASO DEL VOLAPÜK Y DE LA LANGUE BLEUE
}

\author{
José Carlos Martín Camacho \\ Universidad de Extremadura ${ }^{1}$
}

\section{Resumen}

Las lenguas artificiales se emplean para diversas finalidades, entre ellas la de servir como medio de comunicación que elimine las barreras lingüísticas que surgen de la variedad de idiomas existentes en el mundo, algo que permite hablar de un grupo específico de lenguas artificiales, las llamadas lenguas auxiliares internacionales. Estas lenguas auxiliares presentan las mismas propiedades estructurales que las naturales; es decir, una fonología, una gramática y un vocabulario. Por lo que se refiere a la gramática, es de esperar que las lenguas auxiliares alcancen tres rasgos que favorezcan ese objetivo de servir como medio de comunicación entre todos los seres humanos: simplicidad, regularidad y precisión. Este artículo analiza la morfología de dos de estas lenguas, el volapük de Johann Martin Schleyer (1879) y la langue bleue de Lèon Bollack (1900), con el objetivo de determinar el grado en que cumplen esos requisitos.

Palabras clave: Lenguas artificiales, lenguas construidas, lenguas auxiliares internacionales, morfología, volapük, langue bleau.

\section{THE MORPHOLOGY OF ARTIFICIAL LANGUAGES. THE CASES OF VOLAPÜK AND THE LANGUE BLEUE}

\begin{abstract}
Artificial languages are used for several purposes, such as being used as a means of communication that eliminates the linguistic barriers arising from the variety of languages in the world; in this case, specialists speak of international auxiliary languages.
\end{abstract}

1 El presente trabajo se enmarca dentro del proyecto de investigación «En los límites del lenguaje: diseños artificiales y ficciones comunicativas», financiado por el Ministerio de Economía y Competitividad con la referencia FFI2016-76702-P. 
These auxiliary languages have the same structural properties as the natural ones, that is, a phonology, a grammar and a vocabulary. As far as the grammar is concerned, it is to be expected that auxiliary languages will achieve three features that favor that goal of being used as a means of communication among all human beings: simplicity, regularity and precision. This work analyzes the morphology of two of these languages, the volapük by Johann Martin Schleyer (1879) and the langue bleue by Lèon Bollack (1900), with the aim of determining the degree to which they meet these requirements.

Keywords: Artificial Languages, Constructed Languages, International Auxiliary Languages, Morphology, Volapük, Langue Bleue.

\section{Algunos apuntes sobre el complejo mundo de las lenguas artifi- CIALES}

Junto a las lenguas naturales, son muchas las formas de comunicación que el ser humano ha creado para imitar o reemplazar de algún modo esas lenguas, con mayor o menor referencia a ellas y con diversas finalidades. Una forma de comunicación de este tipo, a la que denominaremos lengua artificial, puede definirse como «una construcción semiótica diseñada artificialmente para satisfacer determinados objetivos que las lenguas naturales, constreñidas histórica y culturalmente, no pueden alcanzar» (Galán Rodríguez, 2012: 417).

Estas lenguas artificiales empiezan a tener difusión y relevancia a partir de los siglos XVII y XVIII ${ }^{2}$ (cf. Dodd, 1990: 105-112; Edwards, 2013: 365-366; Pei, 1974: 1001-1002), se han adscrito a muy diversos ámbitos y, a pesar de que tenga cierta extensión la idea de que se reducen a unos cuantos proyectos de iluminados, lo cierto es que han proliferado por doquier y todavía hoy siguen estando muy presentes: no solo el esperanto tiene una difusión digna de respeto, sino que lenguas con fines muy distintos se siguen creando constantemente, como las que se construyen para ficciones artísticas (por ejemplo, el Newspeak de Orwell), las que aún se diseñan para alcanzar el objetivo de proporcionar una lengua que permita la comunicación entre personas diversas (como el europanto, construido en 1996 por Diego Marani, traductor del Consejo de Europa) o las inventadas como lenguas oficiales de las llamadas «micronaciones» que se están extendiendo por el mundo virtual en los últimos tiempos.

No obstante, el estudio que se desarrolla en este trabajo no tiene en cuenta todos estos sistemas lingüísticos, sino solo un grupo muy concreto de ellos, las llamadas lenguas auxiliares internacionales.

2 Sobre los orígenes e historia de estas lenguas y, en general, sobre sus modalidades, objetivos, planteamientos y desarrollos, son muy recomendables el erudito trabajo de Eco (1994) así como el interesante y riguroso ensayo de Galán Rodríguez (2009a). 
Para aclarar este concepto, conviene observar que las lenguas artificiales forman un conjunto de unidades casi innumerable (cf. Eco, 1994: 7-9; Galán Rodríguez, 2009a), se emplean para fines muy diferentes y presentan características diversas en función del modo en que se crean. Por ello, pueden distinguirse dos tipologías de lenguas artificiales.

Por un lado, según su finalidad (cf. Galán Rodríguez, 2009a: 80; Grande Alija, 2001: 31-34; Martínez Gavilán, 2016: 80-82), cabe diferenciar entre:

a) Lenguas creadas para permitir la comunicación entre los seres humanos y la expresión de las ideas. Se incluyen aquí, por una parte, sistemas que han intentado encontrar una forma de transmitir el conocimiento del mundo liberada de los constreñimientos e insuficiencias de las lenguas naturales (entre ellas destacan las llamadas «lenguas filosóficas» de los siglos XVII y XVIII); y, por otra, las lenguas diseñadas para encontrar un medio de comunicación que supere las barreras lingüísticas y, al mismo tiempo, libere de la necesidad de imponer una lengua natural para ese fin. Estas últimas son las llamadas lenguas auxiliares internacionales, como el esperanto o las dos que constituyen el foco central de este trabajo, el volapük y la langue bleue ${ }^{3}$.

b) Un segundo grupo lo constituirían las lenguas creadas como elementos integradores de ficciones, las cuales representan, entre otras posibilidades, la forma de comunicación propia de un lugar o época imaginarios (como el newspeak de 1984), de determinados seres no humanos (p. ej., el klingon de las películas de Star Trek) o de ciertos grupos sociales (caso del nadsat de La naranja mecánica).

Por otro lado, según su forma de construcción, las lenguas artificiales se clasifican en lenguas a priori, lenguas a posteriori y lenguas mixtas (cf. Calero Vaquera, 1999: 11-30; Martínez Gavilán, 2016: 82-83; Pei, 1974: 1007-1011):

a) Las lenguas a priori se construyen sin referencia a las lenguas naturales y con el objetivo de superar las deficiencias propias de estas, de modo que pretenden alcanzar la liberación de todos los idola que impiden a la mente humana aprehender correctamente la realidad (Eco, 1994: 148-160; Galán Rodríguez, 2009b: 106-108). Este grupo está formado principalmente por las ya citadas lenguas filosóficas.

b) Las lenguas a posteriori se crean a partir de las lenguas naturales, de las que toman sus radicales, sea de un conjunto de lenguas distintas o

3 El Diccionario de la lengua española de la RAE incluye entre sus entradas la voz volapuk, con grafía adaptada al español. Sin embargo, preferimos mantener la forma gráfica original porque es la que se emplea de manera general en la bibliografía sobre lenguas artificiales. Por esa misma razón, tampoco traduciremos el nombre de la otra lengua estudiada. 
sea de una sola lengua, tanto antiguas (latín, griego...) como modernas, en cuyo caso se suele acudir a las principales lenguas occidentales (Galán Rodríguez, 2009b: 123; Calero Vaquera, 1990: 32-34). Entre las formadas a partir de varias lenguas, destaca el esperanto; de las basadas principalmente en una sola lengua, cabe citar el latino sine flexione.

c) Finalmente, lenguas mixtas serían aquellas que toman su vocabulario de raíces de las lenguas naturales (como los sistemas a posteriori) pero las deforman hasta tal punto que no se asemejan a ninguna lengua natural y, además, muestran una clara arbitrariedad en la formación de las palabras, en la flexión y en las reglas gramaticales, de modo que recuerdan a los sistemas a priori (Couturat y Leau, 1903: 234-237).

Sin embargo, como han señalado diversos autores (cf. Calero Vaquera, 1999: 27-28; Libert, 2003: 1), el concepto de lengua mixta, grupo al que a menudo se han adscrito las lenguas aquí estudiadas, no resulta muy sólido, pues todas las lenguas artificiales tienen algo de a priori y algo de a posteriori, aparte de que las calificadas como mixtas pueden considerarse en realidad lenguas a posteriori en las que no es sencillo rastrear el influjo de las lenguas naturales.

Partiendo de ahí, se considerará que las lenguas analizadas en este estudio son a posteriori y pertenecen al grupo de las lenguas auxiliares internacionales, esto es, aquellas que buscan servir como medio de comunicación válido para toda la humanidad o, cuando menos, para grupos de personas separadas por la diversidad de lenguas.

Ahondando en lo anterior, cabe señalar que, por lo general, a los creadores de estas lenguas les mueven deseos pragmáticos y filantrópicos, de modo que su fin último al crear una de estas lenguas auxiliares es proporcionar un medio de comunicación que, sin reemplazar las lenguas nativas (Calero Vaquera, 1999: 103; Edwards, 2013: 374), consiga superar las barreras lingüísticas sin necesidad de recurrir a la solución de difundir, o imponer, una lengua natural, ya que ello conlleva evidentes problemas sociopolíticos (cf. Grande Alija, 2001: 32).

Por lo tanto, las lenguas auxiliares internacionales se han presentado como la principal alternativa al uso de lenguas naturales en la comunicación internacional (vid. Comrie, 1996; Pei, 1974: 1002-1003). Pero lo cierto es que, a pesar de que estas lenguas han tenido fervientes defensores, en la actualidad suele afirmarse que han perdido esa especie de batalla contra las lenguas naturales —o, más concretamente, contra el inglés—, de modo que su historia se concibe a menudo como la de un fracaso (Eco, 1994: 19; Calero Vaquera, 1999: 34-37; Grande Alija, 2001: 30-32), fracaso que tiene varias causas: por ejemplo, según Dodd (1990: 126-127), no muchos desean 
aprender una lengua minoritaria y sin cultura propia; estas lenguas son más fáciles de asimilar para los hablantes de las lenguas naturales en las que se basan (normalmente, los occidentales); las lenguas artificiales no tienen en cuenta que las naturales proporcionan diversas visiones del mundo y muchas de ellas muestran cierto fanatismo místico-religioso ${ }^{4}$.

En todo caso, las lenguas artificiales, en especial las auxiliares, no son tan diferentes - en cuanto a construcción estructural se refiere- de las lenguas naturales (Libert, 2003: 2), dado que se han diseñado sobre la base que estas proporcionan y con sus mismos módulos y elementos, pero intentando simplificar y racionalizar la gramática (Eco, 1994: 2018) y el vocabulario. En ese sentido, resumiendo las afirmaciones de autores como Monnerot-Dumanie (1960: 63-67), puede decirse que las lenguas auxiliares internacionales han buscado: la clasificación lógica de las ideas, la neutralidad respecto de las lenguas naturales (si bien esta no siempre se alcanza), la precisión no exenta de riqueza de posibilidades expresivas, la regularidad y la concisión gramatical. En pocas palabras, la simplicidad, la sistematicidad y la precisión. No obstante, estos rasgos deben considerarse más una aspiración que una realidad, pues, como se intentará mostrar mediante el análisis de la morfología del volapük y de la langue bleue, no siempre se alcanzan dichos objetivos.

\section{LENGUAS ARTIFICIALES Y MORFOLOGíA}

Tal como se acaba de señalar, las lenguas auxiliares internacionales - debido a su carácter a posteriori- no difieren estructuralmente de las naturales pues, como ellas, están dotadas de sus mismos niveles y de elementos semejantes; esto es, de pronunciación, de gramática y de léxico. Sin embargo, por lo que respecta a la gramática, sus diseñadores parecen haber pensado, a la hora de construirlas, mucho más en su morfología que en su sintaxis, dado que esta última suele describirse de forma muy somera y se ajusta casi sin divergencias a la de las principales lenguas occidentales ${ }^{5}$.

Pero antes de continuar, conviene concretar qué se entenderá en este trabajo por morfología. En ese sentido, frente a propuestas que asignan a este módulo lingüístico y a la disciplina encargada de su estudio exclusivamente la cuestión de la estructura interna de la palabra, aquí se asumirá

4 Galán Rodríguez (2012: 440) señala, desde una perspectiva más formal, que estas lenguas exigen un considerable esfuerzo de aprendizaje y que, en lugar de contribuir a solucionar el problema de la comunicación internacional, sus creadores parecen más interesados «en las lenguas como manifestación de las operaciones mentales del ser humano que en las lenguas en sí mismas».

5 Esto es, a las que forman el grupo que Whorf calificó como Standard Average European. Vid. al respecto la obra de Libert (2003), en la que se dedican casi sesenta páginas a la morfología (32-89) y poco más de diez a la sintaxis (90-101). 
una perspectiva más amplia que atribuye a la morfología tres campos de estudio interrelacionados ${ }^{6}$ :

a) El estudio del morfema, ámbito en el que habrá que definir esta unidad, establecer su tipología y estudiar los fenómenos derivados de la concreción material de los morfemas en morfos.

b) El análisis de los mecanismos de los que se valen las lenguas para unir morfemas, ya sea con el fin de crear nuevas palabras (morfología léxica o derivativa) o con el de construir la variantes de una misma palabra en función de sus propiedades gramaticales (morfología flexiva).

c) El estudio de la palabra y de sus clases.

Todos estos campos serán objeto de interés en este estudio. Por un lado, se consignarán y analizarán los morfemas que usan las lenguas estudiadas para la formación de nuevas unidades léxicas y para la flexión de las palabras. Por otro, se verá qué clases de palabras se establecen en ellas.

Pero antes de realizar estos análisis, conviene recordar que, según su comportamiento morfológico (en especial en lo que respecta a la flexión), las lenguas se clasifican, desde la lingüística histórico-comparada del xıx, en cuatro grandes grupos (cf. Petersen de Piñeros, 1988: 23-24)":

a) Por un lado, se consideran aislantes o analíticas aquellas lenguas en las que todas las palabras son invariables, esto es, están formadas por un único morfema, de modo que los contenidos gramaticales se expresan mediante recursos como el empleo de partículas. Ejemplo de este grupo son las lenguas chinas.

Frente a ellas, las demás lenguas son sintéticas, dado que en ellas las palabras están —o pueden estar-compuestas por un conjunto de morfemas.

6 Por tanto, frente al tratamiento de autores como Booij (2007) o Matthews (1980), se asumen aquí las propuestas de otros como Bosque (1982), González Calvo (1988), Díaz Hormigo (2003) y Fernández Pérez (1993). El tercero de los campos citados es el que más discusiones produce entre los especialistas, pues la caracterización completa de las clases de palabras necesita del concurso de informaciones procedentes de la sintaxis, aunque es cierto que también puede abordarse exclusivamente desde la morfología. En todo caso, no es este el lugar para debatir la caracterización de la morfología. Únicamente se ha elegido una propuesta teórica que permite abarcar más elementos de estudio y que, de hecho, coincide con el planteamiento de los principales estudios sobre la morfología de las lenguas artificiales, como los de Couturat y Leau (1903), Monnerot-Dumaine (1960) y Libert (2003), que son prácticamente los únicos que desarrollan esta cuestión.

7 Esta clasificación no debe asumirse de forma estricta, sino más bien como un marco conceptual que define unos modelos a los que cada lengua concreta tiende en mayor o menor medida, de modo que una misma lengua puede presentar rasgos de más de un tipo (Robins, 1995: 544-545). 
Según cómo se unen las raíces y los afijos flexivos, dentro de este grupo se distingue entre:

b) Lenguas aglutinantes: se caracterizan por que las palabras están compuestas por secuencias de morfos, cada uno de los cuales representa un único morfema, se sitúa en una posición fija y mantiene los límites respecto de los demás morfos. Ejemplo prototípico de esta clase es el turco.

c) Lenguas flexionales, en las que las palabras están formadas por raíces a las que se unen afijos flexivos que no necesariamente corresponden a un único morfema, de modo que son frecuentes la amalgama y el sincretismo ${ }^{8}$. Entre las lenguas de este tipo se hallan las románicas.

d) Lenguas polisintéticas, cuyo rasgo fundamental es que incorporan a la raíz afijos flexivos que expresan contenidos que en otras lenguas se manifiestan mediante raíces. Ello ocurre en muchas lenguas amerindias.

Respecto de esta clasificación, la situación de las lenguas artificiales ofrece aspectos dignos de estudio que se comentarán en los próximos apartados: por un lado, ha habido autores (Dodd 1960: 125-127; Grande Alija, 2001: 48-50) que han afirmado que lo ideal sería que las lenguas auxiliares siguieran el modelo aislante; pero, por otro, la lingüística histórico-comparada del xix consideró el flexional como el tipo perfecto porque era el más evolucionado (Petersen de Piñeros, 1988: 24; Galán Rodríguez, 2009: 83-85), mientras que las lenguas artificiales tienden por lo general al patrón aglutinante (Galán Rodríguez, 2009: 91).

Con estos rudimentos, se describirá y analizará la morfología de las dos lenguas artificiales que constituyen el foco de este estudio, el volapük y la langue bleue.

\section{El volaPÜK}

\subsection{Introducción}

Inventado en 1879 por el sacerdote y políglota alemán Johann Martin Schleyer, el volapük es la primera lengua a posteriori de relevancia y la pionera del movimiento filantrópico que, a fines del siglo xix y principios del xx, se propuso crear lenguas auxiliares válidas para toda la humanidad ${ }^{9}$.

\footnotetext{
8 Aunque estos dos fenómenos tienen como resultado la aparición en un solo morfo de contenidos que en otros casos se expresan mediante morfemas distintos, el proceso es diferente: la amalgama resulta de una fusión fónica (tipo j’ai en francés), mientras que el sincretismo es producto de la evolución (caso de cant-o en español, donde el morfo -o representa los contenidos que en otras formas verbales aparecen separados).

9 La reseña histórica del volapük se ha elaborado a partir de Couturat y Leau (1903: 128-129; 141-152), Kamman (1942: 189-190), Dodd (1990: 113-114), Libert (2003: 8-9) y Galán
} 
El volapük alcanzó un vertiginoso éxito en el mundo occidental, pero decayó aún más rápido ${ }^{10}$, debido a dos cuestiones interrelacionadas: desde el punto de vista lingüístico, se mostró pronto como una lengua muy compleja, como se ilustrará con detalle en las páginas siguientes; en relación con lo anterior, la intransigencia de Schleyer le llevó a rechazar cualquier propuesta de reforma encaminada a aliviar esa complejidad (Couturat y Leau, 1903: 151-152). Debido a ello, el volapük originó muchas lenguas derivadas (cf. Libert, 2003; Couturat y Leau, 1903) en las que, aun manteniendo la misma base, se intenta encontrar una gramática más simple. Además, la manejabilidad del esperanto hizo que muchos de los primeros seguidores del volapük optaran por la lengua de Zamenhof, creada solo ocho años después que la de Schleyer.

\subsection{Los morfemas del Volapük}

El volapük, como cualquier lengua natural sintética, posee morfemas derivativos y flexivos (cf. Libert, 2003: 35-42; Monnerot-Dumaine, 1960: 8691; Couturat y Leau, 1903: 130, 135-141). Tales morfemas constituyen, como queda dicho, un sistema muy complejo, en especial los flexivos. Véanse los dos cuadros siguientes:

Cuadro 1. Principales morfemas flexivos del volapük. Elaboración propia

\section{Morfemas de caso (sustantivos)}

Ejemplo: fat 'padre'

- Nominativo: $\varnothing /-s$ (fat/fats)

- Acusativo: -i/-is (fati/fatis)

- Genitivo: -a/-as (fata/fatas)

- Dativo: -e/-es (fate/fates)

- Vocativo: $o$ [partícula independiente] (o fat)

* Los adjetivos reciben estos sufijos si van separados del sustantivo o delante de él; si van tras él, se mantienen invariables. Además, todo adjetivo acaba en -ik, sea primitivo o derivado (cf. cuadro 2)

Rodríguez (2009a: 89). En estas referencias pueden encontrarse más detalles sobre la evolución de esta lengua.

10 Couturat y Leau (1903: 151) la consideraban casi extinta ya en 1903: «Aujourd'hui, le Volapük est à peu près mort. Il ne conserve plus qu'un petit nombre de fidèles». No obstante, todavía hoy esta lengua se mantiene viva, como puede verse en la página web $<$ http://volapük. $\mathrm{com} />$, en la que se encuentran los materiales de los que se han extraído las informaciones que se presentan en los siguientes epígrafes (vid. la sección de bibliografía). 
Cuadro 1. Principales morfemas flexivos del volapük. Elaboración propia (cont.)

\section{Morfemas verbales}

2.1. Persona (se emplean los pronombres personales en función de sujeto convertidos en sufijos):

- 1. ${ }^{\mathrm{a}}$ sing.: $-o b / 1$. $^{\mathrm{a}}$ plur.: $-o b s$

- 2. ${ }^{\text {a }}$ sing: $-o l / 2$. $^{\mathrm{a}}$ plur.: -ols

- 3. ${ }^{\text {a }}$ sing. masc.: -om/3. ${ }^{\text {a }}$ plur. masc.: -oms

- 3. ${ }^{\mathrm{a}}$ sing. femen.: $-o f / 3$. $^{\mathrm{a}}$ plur. femen.: $-o f s$

- 3. ${ }^{\mathrm{a}}$ sing. neutro: $-o n / 3$. $^{\mathrm{a}}$ plur. neutro: -ons

* El infinitivo se construye con el sufijo -ön

2.2. Tiempo y aspecto:

- Presente: $\varnothing$

- Presente perfecto: $e^{-}$

- Pasado imperfecto: $\ddot{a}$ -

- Pasado perfecto: $i$ -

- Futuro imperfecto: $o^{-}$

- Futuro perfecto: $u$ -

- Futuro respecto del pasado imperfecto: $\ddot{o}$ -

- Futuro respecto del pasado perfecto: $\ddot{u}$ -

2.3. $V o z$

- Activa: $\varnothing$

- Pasiva: pa- (para el presente)/p- (para el resto de formas)

2.4. Modo

- Indicativo: $\varnothing$

- Imperativo: -öd

- Optativo: - ös

- Condicional: - öv

- Subjuntivo - -la (se añade el guion al construir la forma)

Ejemplos: löfön 'querer'

a) Presente de indicativo en voz activa:

- Singular: löfob, löfol, löfom/löfof/löfon

- Plural: löfobs, löfols, löfoms/löfofs/löfons

b) Pasado imperfecto de indicativo en voz activa

- Singular: älöfob, älöfol, älöfom/älöfof/älöfon

- Plural: älöfobs, älöfols, älöfoms/älöfofs/älöfons

c) Presente perfecto de indicativo en voz pasiva:

- Singular: pelöfob, pelöfol, pelöfom/pelöfof/pelöfon

- Plural: pelöfobs, pelöfols, pelöfoms/pelöfofs/pelöfons

d) Presente de subjuntivo en voz activa:

- Singular: löfob-la, löfol-la, löfom-la/löfof-la/löfon-la

- Plural: löfobs-la, löfols-la, löfoms-la/löfofs-la/löfons-la 
Cuadro 2. Morfemas derivativos del volapük. Elaboración propia

\section{Prefijos}

- al- 'cada'. Ej.: aldelik 'diariamente' < del 'día'.

- beno- 'bien'. Ej.: benosek 'éxito' < sek 'suceso, hecho'.

- dä- 'en pedazos'. Ej.: däbreikön 'romper en pedazos' < breikön 'romper'.

- dei- 'hasta la muerte'. Ej.: deibludön 'morir desangrado' < bludön 'sangrar'.

- do- 'movimiento hacia abajo'. Ej.: docöpön 'tronchar' < cöpön 'picar'.

- fa- 'ausencia'. Ej.: fabinön 'ausente' < binön 'ser'.

- fäi- 'cerrado'. Ej.: fäitanön 'vendar' < tanön 'atar'.

- fe- 'consumo completo'. Ej.: fepledön 'perder en el juego' < pledön 'jugar'.

- fi- 'hasta el fin'. Ej.: fimekön 'completar' < mekön 'hacer'.

- ge- 'retorno'. Ej.: gegivön 'devolver' < givön 'dar'.

- hi- 'macho, masculino'. Ej.: himatan 'esposo' < matan 'cónyuge'

- ho- 'sin masculinidad'. Ej.: hojevod 'caballo castrado' < jevod 'caballo'

- ji- 'hembra, femenino'. Ej.: jimatan 'esposa' < matan 'cónyuge'.

- jo- 'sin feminidad'. Ej.: jogok 'gallina castrada' < gok 'gallina'.

- ke- 'junto, unión'. Ej.: kelied 'compasión' < liedön 'sufrir'.

- la- 'levantando'. Ej.: lasumön 'levantar' < sumön 'tomar'.

- läx- 'ex-, emérito'. Ej.: läxprofäsor 'ex profesor' < profäsor.

- le- (aumentativo). Ej. ledom 'mansión' < dom 'casa'.

- lu- (despectivo). Ej. ludom 'choza' < dom 'casa'.

- lü- 'por ley (parentesco)'. Ej.: lüfat 'suegro' < fat 'padre'.

- mi- 'malo, nocivo'. Ej.: mikeb 'maleza' < keb 'hierba'.

- ne- 'contrariedad'. Ej.: neflen 'enemigo' < flen 'amigo'.

- ni- 'interior'. Ej.: niklots 'ropa interior' < klots 'ropa'.

- nü- 'movimiento hacia adentro'. Ej.: nübladön 'inspirar' < bladön 'soplar'.

- plö- 'exterior'. Ej.: plögun 'abrigo' < gun 'chaqueta'.

- ru- 'primitivo, antiguo'. Ej.: rutim 'prehistoria' < tim 'tiempo'.

- sä- 'remisión'. Ej.: säkusad 'excusa' < kusadön 'acusar'.

\section{Sufijos}

- -af 'nombres de animales'. Ej.: sugaf 'mamífero' < sug 'succión'.

- -ag 'abundancia'. Ej.: herag 'peludo' < her 'pelo'.

- -am 'acción de'. Ej.: penam 'escritura' < penön 'escribir'.

- -an 'alguien que es o hace'. Ej.: tidan 'maestro' < tidön 'enseñar'.

- -at 'cantidad de'. Ej.: degat 'decena' < deg 'diez'.

- -av 'ciencia'. Ej.: kalkulav 'aritmética' < kalkulön 'calcular'.

- -äb 'receptor, dativo'. Ej.: fanäb 'prisionero' < fanön 'aprisionar'.

- -äd 'efecto de'. Ej.: spikäd 'habla' < spikön 'hablar'.

- -än 'país'. Ej.: Linglän = Inglaterra.

- -ät 'abstracción'. Ej.: kompenät 'compañía' < kompenön 'compartir'.

- -ed 'particularización'. Ej.: pened 'letra' < penön 'escribir'.

- -ef 'conjunto de personas'. Ej.: menef 'humanidad' < men 'hombre'.

- -el 'fabricante de'. Ej.: bodel 'panadero' < bod 'pan'.

- -em 'conjunto de cosas'. Ej.: bledem 'follaje' < bled 'hoja'. 
Cuadro 2. Morfemas derivativos del volapük. Elaboración propia (cont.)

- -ep 'planta, árbol'. Ej.: bünep 'peral' < bün 'pera'.

- -er 'que contiene'. Ej.: feriner 'ferroso' < ferin 'hierro'.

- -et 'resultado de, producto de'. Ej.: penet 'nota, noticia' < penön 'escribir'.

- -iäl 'tendencia, inclinación'. Ej.: okiäl 'egoísmo' < ok 'uno mismo'.

- -iär 'recipiente'. Ej.: nigiär 'tintero' < nig 'tinta'.

- -ik (adjetivador). Ej.: dibik 'profundo' < dib 'profundidad'.

- -il (diminutivo). Ej.: dogil 'perrito' < dog 'perro'.

- -im 'filosofía, doctrina'. Ej.: tikälim 'racionalismo' < tikäl 'razón'.

- -o (adverbializador). Ej.: boniko 'buenamente' < bonik 'bueno'.

- -od 'ejemplo menos serio'. Ej.: magod 'pintura' < magön 'pintar'.

- -ot 'ejemplo más serio'. Ej.: magot 'estatua' < magön 'pintar'.

- -ov 'posibilidad'. Ej.: pronov 'pronunciable' < pronön 'pronunciar'.

- -ö 'cualidad'. Ej.: manöf 'virilidad' < man 'hombre'.

- -ön (verbalizador). Ej.: debön 'adeudar' < deb 'deuda'.

- -öm 'aparato'. Ej.: flitöm 'avión' < flitön 'volar'.

- -öp 'lugar'. Ej.: potöp 'oficina de correos' < potön 'enviar por correo'.

- -ül ‘joven, cría'. Ej.: dogül 'cachorro' < dog 'perro'.

\subsubsection{Observaciones generales}

Del análisis de los cuadros anteriores pueden extraerse varios rasgos globales de la morfología del volapük:

a) Es una lengua claramente aglutinante, pues en ella se cumple la propiedad de que los morfos aportan un solo contenido y se presentan con un orden fijo. Ello se observa, v. g., en las pautas de creación de las formas verbales (vid. cuadro 1). Por ej., pelöfobs 'hemos sido queridos' está compuesta por los morfos $p$ - 'pasiva', $e$ - 'presente perfecto', löf 'querer', - $o b$ 'primera persona' y $-s$ 'plural'. Como se ve, la diferencia entre esta forma y la propia del español es enorme, pues en una sola palabra fónica se condensa lo que en español se expresa en tres. Este comportamiento tiene la ventaja, respecto de las lenguas flexionales, de que ofrece una pauta de formación mecánica, pero, como contrapartida, obliga a construir cada forma con muchas operaciones mentales, frente al empleo de paradigmas propio de las lenguas flexionales.

b) Es una lengua que solo presenta tres componentes morfológicos: prefijos, sufijos y raíces. Ello supone una simplificación respecto de la mayor parte de las lenguas naturales, que junto con esos tres tipos básicos suelen tener algún otro como la circunfijación o la infijación. Por tanto, en esto es una morfología sencilla, pero, si de simplificar se trata, habría que observar que en realidad el empleo de prefijos y sufijos no se justifica, 
pues ambos aportan funcionalidades similares, tanto en la flexión como en la derivación ${ }^{11}$.

c) Cada uno de esos tres formantes morfológicos presenta un contorno fonológico definido: salvo excepciones, los prefijos acaban en vocal, las raíces comienzan y terminan por consonante, y los sufijos empiezan por vocal. Por ej., o-löf-ob 'yo querré', fi-mek-ön 'completar', tid-an 'maestro'. Ello evita la formación de los problemáticos procesos morfonológicos que afectan a las lenguas naturales: por ej., en español, ¿contraatacar o contratacar?; irreductible, no *inreducible.

d) Igualmente, los morfemas carecen de la alomorfia propia de cualquier lengua natural, lo cual facilita el uso de los afijos, dado que no se producen dualidades ni condicionamientos externos a su empleo.

\subsubsection{Sobre los morfemas flexivos}

Profundizando en el análisis de la flexión, hay que destacar que Schleyer planificó esta lengua intentando cubrir gran cantidad de contenidos gramaticales mediante recursos flexivos, algo que provoca una enorme complejidad y contradice la sencillez que la lengua muestra en otros aspectos:

a) La flexión casual del sustantivo no simplifica, sino que complica el sistema; de hecho, evolutivamente la mayor parte de las lenguas modernas la han cambiado por el empleo de preposiciones. Pero es que, además, el volapük permite en algunos casos alternar preposiciones y flexión casual, algo que aumenta la dificultad al duplicar la forma de manifestar un mismo contenido.

Igualmente, cabe comentar la situación de los adjetivos: en principio, se recomienda que sigan al sustantivo, posición en la que no llevan terminaciones de caso; pero, si por motivos estilísticos, el adjetivo precede al sustantivo o queda separado de él por otra palabra, debe llevar las mismas terminaciones que este. Obviamente, esta duplicidad aumenta la complejidad de uso de la lengua y, además, introduce un factor, el estilístico, que no cuadra con los objetivos utilitarios de las lenguas auxiliares.

b) Pero mucho más complicado es el sistema verbal, en el que se expresan morfemáticamente gran cantidad de contenidos: en los cuadros se consignan persona, tiempo-aspecto, modo y voz, cada uno de los cuales a su vez ofrece varias posibilidades; pero también existe, por ejemplo, la posibilidad de emplear elementos para hacer transitivo un verbo intransitivo o a la inversa. Ello determina una complejidad que se manifiesta en dos hechos:

11 Ciertamente, esta dualidad de empleo de distintos tipos de afijos para funciones similares es algo más que común en las lenguas naturales, pero si el objetivo es simplificar al máximo, el ideal sería utilizar solo un tipo de afijo. 
Por un lado, se hacen distinciones poco rentables. Por ejemplo, el modo optativo es solo una variante suavizada del imperativo en la que se da al oyente la opción (de ahí el nombre) de realizar o no lo que se le ordena. Evidentemente, es más simple usar una fórmula fija para expresar este matiz (si no te/le importa, por favor...) que crear formas flexivas diferenciadas. De igual modo, se emplea la terminación -ikön para verbos intransitivos, la cual se cambia por -ükon si se quiere hacer transitivo el verbo: finikön es la forma intransitiva de 'terminar' y finükön la transitiva, algo que parece ocioso, pues la construcción sintáctica desambigua este tipo de verbos: La función terminó pronto/El empleado terminó sus tareas.

Por otro lado, se usan morfemas para construcciones que en muchas lenguas se expresan perifrásticamente, como el condicional, que en idiomas como el inglés se construye mediante la perífrasis con would, o la pasiva, que en español es una construcción sintáctica (ser+participio).

En definitiva, la morfología flexiva del volapük, y en especial la verbal, resulta muy complicada, tanto por la cantidad de contenidos que expresa como por su sistema aglutinante (cf. Dodd, 1990: 114, quien afirma que el volapük padece de hipertrofia) ${ }^{12}$.

\subsubsection{Sobre los morfemas derivativos}

Los morfemas propuestos para la creación de palabras manifiestan un intento de simplificar el comportamiento propio de las lenguas naturales que, no obstante, no siempre se consigue. Varios son los aspectos dignos de comentario $^{13}$ :

a) Son pocos elementos: según las fuentes empleadas, 25 prefijos y 31 sufijos. En español, aunque los listados varían mucho de unos autores a otros, un cómputo personal utilizado para otra investigación dio como resultado 47 prefijos y 81 sufijos. Se trata, pues, de una reducción cuantitativa evidente que, en principio, contribuye a la simplificación aludida, dado que:

Para la transcategorización se usan elementos únicos que, además, son los propios morfemas flexivos. Así, hay un solo sufijo verbalizador, que además es el morfema flexivo de infinitivo (-ön), frente al español, que emplea para este fin tanto el flexivo -ar como los derivativos -e-ar, -ec-er, -iz-ar e -ific-ar.

12 Comrie (1996: 44-45) comenta que una lengua artificial que hiciera todas las distinciones gramaticales posibles sería una lengua inutilizable, y el volapük no parece lejos de ese límite. Véanse las críticas que en el mismo sentido hacen Couturat y Leau (1903: 141-152 y 153-159), quienes afirman que Schleyer quiso dar a su lengua todos los recursos que pueden ofrecer las lenguas vivas con el fin de dotarla de los instrumentos para traducir los matices más complejos y sutiles del pensamiento, mientras que los partidarios de las lenguas auxiliares lo que suelen buscar es una lengua simple y eficiente.

${ }_{13}$ En todo este apartado emplearemos el español como base para las comparaciones. 
También hay un solo adjetivador, $-i k$, que es la terminación de todos los adjetivos, mientras que en español compiten formas como -al (otoñal), -ario (bancario), -able (adorable)... Y solo hay un adverbializador (-o), algo en lo que el español se comporta igual, pues solo tiene -mente.

Prácticamente no hay sinonimia ni polisemia. Respecto de la sinonimia, no se localizan (cf. cuadro 2) prefijos o sufijos distintos con igual contenido, algo frecuente en español. Así, mientras que en volapük solo hay tres apreciativos, uno diminutivo (-il), otro aumentativo (le-) y otro despectivo (lu-), en español hay varios sufijos de cada uno de estos tipos (-ito, -illo, -ino, -uelo; -ote, -azo, -ón; -ujo, -uco, -uzo $)^{14}$. Respecto de la polisemia, tampoco parece haber morfemas con más de un contenido, algo habitual en las lenguas naturales: por ej., en español -ero expresa 'agente' (camionero), 'lugar' (basurero), 'relación' (barriobajero) y gentilicio (lepero). Solo se detecta en volapük un sufijo que, más que polisemia, presenta un contenido vago: -an, que según las fuentes se define como 'alguien que es o hace algo' o como 'miembro de una profesión, asociación, país, doctrina o disciplina'.

b) Con sus afijos derivativos, el volapük expresa contenidos habituales en las lenguas naturales: no faltan, entre otros, los que indican 'contrariedad' (ne-), 'lugar' (-öp), 'recipiente' (-iär) o 'agente' (-an). Pero también se emplean otros de significado muy preciso: por ej., la pareja ho-/jo('sin masculinidad'/'sin feminidad') o la dualidad -ef/-em ('conjunto de personas'/'conjunto de cosas'). Al respecto, hay que recordar que las lenguas naturales codifican a menudo de forma caprichosa los contenidos derivativos (por ej., en español un sufijo como -izo, unido a adjetivos, expresa un contenido muy especial: 'con propiedades de X, pero sin llegar a ser X'), pero lo cierto es que, en nuestra opinión, una lengua que busque la simplicidad debería limitarse a contenidos más genéricos.

c) Finalmente, aunque esta es una afirmación impresionista, parece que estos afijos derivativos ofrecen poca productividad. De hecho, de las fuentes consultadas se infiere que estos afijos se usaron ad hoc para crear el vocabulario inicial de la lengua y después han sido utilizados pocas veces: haciendo una breve cala, se ha comprobado que el volapük ha habilitado palabras para referirse al mundo informático, y lo ha hecho a veces con morfemas derivativos (por ej., monitor es jonülöm, que deriva de jonülön 'mostrar' con el sufijo -öm 'aparato'; ordenador es nünöm, derivado de nüm 'número'). Pero la impresión global es que se trata de casos aislados, pues la lengua parece preferir el préstamo o la adaptación para cubrir las necesidades que van

14 Pero obsérvese la inconsistencia de expresar la disminución con sufijo y el aumento y el desprecio con prefijos, cuando los tres tipos de contenidos pertenecen a una misma esfera conceptual (la expresión del tamaño y de la valoración apreciativa). 
apareciendo: el ratón se nombra como mugaparat 'aparato ratón' o hacker como nüdranädan 'entrometido'.

En resumen, los afijos derivativos del volapük buscan la simplicidad, algo en lo que se «mejora» el funcionamiento de las lenguas naturales, pero no siempre se consigue, pues algunos elementos tienen un contenido muy concreto y su productividad parece limitada.

\subsection{Las clases de palabras del volapük}

El volapük distingue las siguientes clases de palabras (cf. Libert, 2003: 43-89; Couturat y Leau, 1903: 129-135): sustantivos, adjetivos, verbos, adverbios, pronombres (personales y otros que se emplean también como determinantes; en concreto, posesivos, demostrativos, indefinidos, interrogativos, exclamativos y relativos), interjecciones, preposiciones y conjunciones.

Por tanto, en este ámbito la única peculiaridad del volapük es la ausencia de artículos, palabras que según sus gramáticas no resultan necesarias.

\subsection{Corolario}

De lo expuesto se deduce que la morfología del volapük sigue muy de cerca la de las lenguas naturales, de modo que podría funcionar como cualquiera de ellas ${ }^{15}$. Sin embargo, el haber creado una lengua planificada determina que se presenten diferencias respecto de las lenguas naturales que conviene notar. Esas diferencias se resumen en el hecho de que, aunque parece que la intención primigenia de Schleyer era simplificar lo que ofrecen las lenguas naturales (de ahí que no haya alomorfia, sinonimia, polisemia, fenómenos morfonológicos...), ello no siempre se consigue. Esto se observa especialmente en la morfología flexiva, que concentra con medios morfemáticos muchos contenidos diversos y cuya razón de ser a menudo no se justifica desde el punto de vista del uso de los afijos para tales fines; dicho de otro modo, la flexión acoge muchos contenidos que podrían expresarse con medios más económicos. Por su parte, la morfología derivativa es interesante, pero no parece muy productiva y el número de sus unidades es reducido.

\section{LA LANGUe BLeUe}

\subsection{Introducción}

La langue bleue es otro de los proyectos de lengua auxiliar surgidos a fines del xıx y principios del xx, si bien, frente al volapük, nunca tuvo, si puede

15 Esta afirmación no es baladí, pues sería muy discutible si los sistemas a priori ofrecen esta posibilidad. No obstante, ello no es materia de este estudio. 
decirse así, más practicantes que su propio creador, el comerciante francés Lèon Bollack, quien, al no conseguir adeptos para su lengua, se hizo seguidor del ido. Como el volapük, es una lengua basada fundamentalmente en las románicas y germánicas (cf. Libert, 2003: 2-3; Couturat y Leau, 1903: 210).

Según afirma el propio diseñador en el prefacio de su Grammaire abrégée de la langue bleue (Bollack, 1900: I-IV), fuente del análisis que se expone seguidamente, su proyecto cumple con lo que se debe pedir a un idioma que quiera servir como segunda lengua a toda la humanidad: concisión, claridad, precisión y sistematicidad. El siguiente análisis tratará de confirmar o negar esa afirmación.

\subsection{Los morfemas de la langue bleue}

Como en el caso anterior, se presentarán en sendos cuadros los morfemas flexivos y derivativos de la langue bleue (cf. las informaciones de Libert, 2003: 35-42; Monnerot-Dumaine, 1960: 82-83; Couturat y Leau, 1903: 221-224).

\section{Cuadro 3. Principales morfemas flexivos de la langue bleue. Elaboración propia}

\section{Morfemas de caso}

Se aplican a los pronombres personales, relativos e indefinidos (vid. ejemplo de conjugación).

\section{Morfemas nominales}

- -u 'plural'. Por ej.: bov 'toro' - bovu 'toros'.

- u- 'femenino'. Solo para seres vivos, con el fin de diferenciar masculino de femenino; los demás sustantivos son neutros. Por ej.: bov 'toro' - ubov 'vaca'.

\section{Morfemas verbales}

\subsection{Persona}

No se expresa con morfemas, porque se considera suficiente el sujeto (nombre o pronombre). La impersonalidad se expresa con el pronombre de tercera persona neutro.

\subsection{Modo}

Se distinguen cuatro modos, ninguno de los cuales tiene expresión morfemática:

- Infinitivo: se manifiesta en que no lleva ninguna palabra que funcione como sujeto.

- Indicativo: lleva un sujeto en nominativo.

- Exclamativo (o imperativo): lleva un sujeto en vocativo.

- Subordinado (o subjuntivo): se introduce con una palabra-marco entre las dos proposiciones; por ej., $k u$. 
Cuadro 3. Principales morfemas flexivos de la langue bleue. Elaboración propia (cont.)

3.3. Tiempo y aspecto

A) Cada modo tiene cuatro tiempos, que se expresan con sufijos:

- Presente: -o.

- Pasado: -e.

- Futuro: -a.

- Eterno: -i.

B) El aspecto solo se aplica al modo indicativo y se expresa mediante el prefijo $u$ - en el caso de la anterioridad.

3.4. Voz

Existen tres voces:

- Activa: no lleva ninguna marca

- Pasiva: se expresa con un sufijo - $u$, que se sitúa delante del sufijo de tiempo.

- Reflexiva: se expresa con la partícula su, si es afirmativa, y con snu si es negativa. Ejemplos: lovi 'amar'

a) Presente de indicativo en voz activa:

- Singular: me lovo, te/vos lovo, se/le/ye lo lovo.

- Plural: ne lovo, pe/ge lovo, be/fe/de lovo.

b) Pasado imperfecto de indicativo en voz activa:

- Singular: me love, te/vos love, se/le/ye love.

- Plural: ne love, pe/ge love, be/fe/de love.

c) Presente perfecto de indicativo en voz pasiva:

- Singular: me ulovuo, te/vos ulovuo, se/le/ye ulovuo.

- Plural: ne ulovuo, pe/ge ulovuo, be/fe/de ulovuo.

d) Presente de subjuntivo en voz activa:

- Singular: ku me lovo, ku te/vos lovo, ku se/le/ye lovo.

- Plural: ku ne lovo, ku pe/ge lovo, ku be/fe/de lovo.

Cuadro 4. Morfemas derivativos de la langue bleue. Elaboración propia

\section{Prefijos}

\subsection{Interjecciones ( $\left.a-, e_{-}, i-, o^{-}\right)$}

Prefijadas, estas interjecciones expresan 'desaliento', 'aprobación', 'alegría' y ‘duda'.

1.2. Palabras-marco: algunas son prefijos que expresan un contenido sistemático:

- ru- 'repetición'.

- bu- 'superioridad'.

- pu- 'supremacía'.

- yu- 'inferioridad'.

- flu- 'contrariedad'.

- plu- 'pluralidad, conjunto'.

- pru- 'preexistencia'.

- sku- 'semejanza'.

- pru- 'promoción'.

- pnu- 'diferencia'.

16 El signo $\psi$, según Bollack, representa el sonido que tiene en francés la grafía $t c h$, esto es, el palatal $\left[\mathrm{t} \int\right]$. 
Cuadro 4. Morfemas derivativos de la langue bleue. Elaboración propia (cont.)

\section{Sufijos}

2.1. Terminaciones absolutas: se emplean sistemáticamente.

A) Transcategorizadores

A partir del sustantivo se forman las otras palabras grandes. Así, de lov 'amor':

- Los verbos se forman con los morfemas flexivos correspondientes: lovi 'amar'

- Los atributivos se forman con la estructura vocal $+d$, del siguiente modo:

- -ad: forma adjetivos con sentido de posibilidad. Ej.: lovad 'digno de amar'.

- -ed: forma adjetivos de sentido general. Ej.: loved 'amado'.

- -id: forma participios de presente de cualidad permanente. Ej.: lovid 'amante (en general)'.

- -od: forma participios de presente de cualidad transitoria. Ej.: lovod 'amante (en un momento dado)'.

- Los modificativos se forman con la estructura vocal +4 , del siguiente modo:

- -ay: forma adverbios de modo con sentido de posibilidad. Ej.: lovay agradablemente'.

- -ey: forma adverbios de modo con sentido general. Ej.: lovey 'con amor'.

- -iц: forma participios de presente de cualidad permanente. Ej.: loviy 'amante (en general)'.

- -oy: forma participios de presente de cualidad transitoria. Ej. lovoy 'amante (en un momento dado)'.

B) Otras terminaciones absolutas

- -in 'esposa de'. Ej.: prinsin 'la esposa del príncipe' < prins 'príncipe'.

- -an 'gentilicio'. Ej.: fransan 'francés'.

2.2. Terminaciones secundarias: no se emplean de forma sistemática ${ }^{17}$

- -adr 'de la naturaleza de, semejanza'. - -ak 'hecho de'.

- -alg 'enfermedad'.

- -ap 'quien aporta algo'.

- -asl 'que odia'.

- -ef 'efecto, resultado de'.

- -erk 'profesión'.

- -et 'diminutivo'.

- -ig 'acción de'.

- -art 'parte de'.

- -as 'aumentativo'.

- -av 'cualidad'.

- -enk 'comienzo de una acción'.

- -est 'jefe'.

- -ibl 'que ama algo'.

- -ik 'ciencia'.

- -il 'instrumento': kotil 'cuchillo' < kot 'corte'.

- -is 'multiplicidad'.

- -it 'destino, uso'.

- -ir 'devenir de una acción o estado'.

- -odr 'descendiente, derivado de'.

- -ist 'trabajador'.

- -iv 'contenedor'.

- -olb 'golpe de'.

- -olm 'árbol después de la flor o el fruto'. - -olv 'lugar plantado de'

- -or 'agente'. Ej.: spilor 'jugador' < spil 'juego'. • -orm 'con forma de'

- -ort 'lugar'. Ej.: panort 'panadería' < pan 'pan'. • -os 'término científico'.

- -osm 'generalidad física'.

- -ost 'fabricante de'.

17 De esta sección no es posible aportar apenas ejemplos porque no se localizan en las fuentes que hemos podido manejar. 


\subsubsection{Observaciones generales}

Según su propio diseñador (Bollack, 1900: I-IV), la langue bleue es un idioma creado con la filantrópica intención de convertirse en una segunda lengua que pueda servir como medio de comunicación neutro para cualquier hablante. Tomando como base esta idea, su objetivo es partir de la observación precisa de los objetos e ideas de la realidad para convertirlos en contenidos lingüísticos y encontrar para ellos formas de expresión concisas, claras, precisas y sistemáticas.

La búsqueda de esos ideales se manifiesta en algunas pautas ya vistas respecto del volapük: la lengua tiende al tipo aglutinante y los morfemas se limitan a tres clases (raíces, sufijos y prefijos), que presentan un contorno fonológico definido y no muestran alomorfia.

Pero volapük y langue bleue son lenguas muy distintas, pues esta última es bastante más sencilla, en especial en la flexión, dado que evita en muchos casos el uso de morfemas, que sustituye por el empleo de palabras y partículas, de ahí que, a pesar de su carácter aglutinante, pueda afirmarse que esta lengua posee también ciertos rasgos de lengua aislante.

\subsubsection{Sobre los morfemas flexivos}

Como se acaba de señalar, lo más destacable de la langue bleue es que, a pesar de su funcionamiento aglutinante, Bollack prefiere, para evitar la expresión de contenidos redundantes, no utilizar morfemas, que sustituye por otros mecanismos, en especial por el uso de partículas y de pronombres ${ }^{18}$. En ese sentido, cabe destacar:

En los verbos, la persona se expresa solo mediante el sujeto, sin ningún elemento flexivo; de igual modo, la impersonalidad se manifiesta con el pronombre de 3 . $^{\mathrm{a}}$ persona neutro; los modos se forman mediante el uso, o ausencia, de determinados pronombres, la anterioridad solo se manifiesta en indicativo... Ello hace que el mecanismo de construcción de las formas verbales sea bastante más sencillo que en volapük (cf. cuadro 3), algo que no es óbice para que los verbos transmitan muchos de los contenidos gramaticales que se dan en las lenguas románicas y germánicas.

Los sustantivos solo varían en cuanto al número, algo casi imprescindible. Respecto del género, únicamente se expresa por medio de desinencias en

18 Como es bien sabido, la repetición de los mismos contenidos morfemáticos en una expresión suele considerarse redundante en las lenguas naturales; por ejemplo, la reiteración del plural y del femenino en una secuencia como Las alumnas aplicadas. 
el caso de los seres vivos ${ }^{19}$, pues todos los demás son neutros y carecen de marca flexiva.

Respecto de los adjetivos, son invariables en cuanto a género y número, dado que esos contenidos ya están expresados en el sustantivo, lo cual permite suprimir la concordancia sustantivo-adjetivo.

La flexión casual no existe, salvo en los pronombres personales, relativos e indefinidos, algo que, no obstante, no queda muy justificado.

En definitiva, y a pesar de la opinión de Couturat y Leau (1903: 225-233), que presentan diversos argumentos para atacar lo que a su juicio es una gramática arbitraria y difícil, parece que el sistema flexivo de la langue bleue consigue en líneas generales la sencillez, precisión y concisión anunciadas por Bollack. De todas formas, quedan algunas lagunas, como el citado uso de la declinación casual en los pronombres o el ambiguo empleo de la vocal $u$, a la que se recurre para expresar diversos contenidos, sea como prefijo o como sufijo (vid. cuadro 3).

\subsubsection{Sobre los morfemas derivativos}

El sistema de morfemas derivativos de la langue bleue se presenta en la obra de Bollack de forma dispersa e incompleta, pero, a pesar de ello, puede considerarse válido y efectivo, algo que se manifiesta en varios hechos:

a) En el empleo de prefijos y sufijos que aportan contenidos sistemáticos (vid. cuadro 4), contenidos que, además, coinciden con los habituales en las lenguas naturales: 'repetición', 'contrariedad', 'gentilicio', 'diminutivo', 'conjunto'... En todo caso, como se dijo respecto del volapük, podría haberse suprimido la distinción entre ambos tipos de afijos, pues en esencia su funcionalidad es la misma.

b) En el sistema de transcategorización diseñado por el autor (vid. cuadro 4), que basa toda la formación del vocabulario en los sustantivos, a partir de los cuales se crean con sufijos las otras clases de palabras principales. Sin embargo, en este punto la exposición de la formación de atributivos y modificativos queda confusa, pues de hecho se presentan en ambos grupos palabras distintas a las que se dan los mismos significados: lovid 'amante (en general)' y lovod 'amante (en un momento dado)' son calificativos; pero también hay dos modificativos con idéntico significado, loviy y lovoy, respectivamente.

19 Ello es un rasgo muy frecuente en las lenguas artificiales, pues resulta obvio que, en términos cualitativos, expresar el género más allá de los seres vivos resulta una complicación gramatical innecesaria (cf. Galán Rodríguez, 2009a: 71; Libert, 2003: 36-37). 
Aparte de ello, hay otros aspectos mejorables, como que considere palabras (aunque sean palabras-marco; cf. \$4.3) los prefijos citados en el epígrafe 1.2 del cuadro 4; que convierta en prefijos las interjecciones, pues estas podrían haberse usado con la misma finalidad como palabras independientes; o que en las llamadas terminaciones secundarias aparezcan contenidos demasiado concretos, como, por ejemplo, 'que odia' o 'árbol después de la flor o el fruto'.

\subsection{Las clases de palabras de la langue bleue}

Quizás lo más original de la morfología de esta lengua sea su sistema de clases de palabras (Bollack, 1900: 10-35). Ese sistema se inspira básicamente en el francés, pero en él se reordenan las categorías y se introducen algunas modificaciones y novedades.

Como punto de partida, Bollack (1900: 7) distingue dos grandes categorías semántico-conceptuales a las que llama nociones vagas y nociones precisas («notions vagues et notions précises»), cada una de las cuales se materializará en tipos específicos de palabras que tendrán una forma diferenciada por su longitud y estructura fonológica. Las nociones vagas expresan relación entre las palabras, mientras que las nociones precisas expresan 'sustancia', 'estado' o 'acción'. Las primeras se manifiestan mediante significantes cortos y originan las motules (que traduciremos por particulas); las segundas se transmiten mediante significantes más largos y dan lugar a granmots (que traduciremos por palabras grandes). A partir de ahí, Bollack propone la siguiente clasificación:

1. Motules:

1.1. Interjecciones.

1.2. Palabras-marco (mots-cadre): es una nueva clase propuesta por el autor que comprende la expresión de ideas generales y de algunos modos de abreviación. En concreto, son partículas que expresan 'negación', 'reflexividad', 'interrogación' y contenidos verbales perifrásticos (por lo que reemplazan a los auxiliares verbales); afijos que expresan ideas globales como 'reiteración' o 'superioridad' y partículas (que él llama abréviatifs) que indican por ejemplo que la palabra que sigue es un nombre propio o es extranjera.

1.3. Conectivos (connectifs): preposiciones y conjunciones.

1.4. Designativos (désignatifs): artículos (solo indeterminados), adjetivos determinativos (salvo los numerales) y pronombres. 


\section{Granmots:}

2.1. Nombres y numerales (ordinales y cardinales), que forman para el autor un mismo grupo.

- Verbos: excepto el participio y el gerundio.

- Atributivos (attributifs): adjetivos y participios.

- Modificativos (modificatifs): adverbios y gerundios.

Por tanto, Bollack se aparta de la clasificación prototípica de la gramática grecolatina heredada por la lingüística europea, redistribuyendo los tipos y subtipos e introduciendo las "palabras-marco», que son una especie de cajón de sastre que engloba muchos contenidos dispares, si bien todos de tipo gramatical y relacional.

Es este un sistema interesante, que manifiesta, entre otras cosas, que el autor percibe la relación que existe, por ejemplo, entre preposiciones y conjunciones, o entre artículos y determinantes, y que ve claramente la diferencia entre contenidos léxicos y gramaticales. Pero también toma decisiones poco justificadas, como considerar palabras elementos que funcionan como afijos o equiparar en un mismo grupo los numerales y los sustantivos.

Por otro lado, cada clase de palabras posee forma propia. Las palabras breves tienen 1, 2 o 3 letras, y si tienen tres acaban en vocal; en cambio, las largas se forman con tres letras o más, y terminan en consonante si son de tres letras. De este modo, cada clase presenta una forma fija: por ej., los sustantivos siempre empiezan y acaban por consonante (de ahí que los sufijos comiencen por vocal y los prefijos acaben en vocal), mientras que los verbos empiezan por consonante y terminan en vocal.

\subsection{Corolario}

De lo expuesto cabe inferir que, al menos desde el punto de vista morfológico, la langue bleue está construida sobre parámetros más simples que los del volapük.

La morfología flexiva es mucho sencilla que la de la lengua de Schleyer. De hecho, mientras que el volapük recuerda a una especie de mezcla del turco con el latín y las lenguas germánicas, la langue bleue se aproxima al ideal de una lengua con "poca gramática», algo que, aunque sin rigor científico, se atribuye a menudo al inglés como causa de su triunfo internacional (cf. Simone, 1992: 166-167).

Por su parte, en la morfología derivativa las diferencias son menores, aunque parece que la langue bleue presenta más rigor que el volapük, si bien quedaría por ver la funcionalidad de los morfemas derivativos - en especial, la de los sufijos que Bollack llama «terminaciones secundarias»-, pues su 
establecimiento, al menos en la gramática consultada, no pasa de ser un esbozo (no hemos logrado acceder al diccionario de la lengua que el autor publicó dos años después de la gramática, por lo que desconocemos qué productividad «real» tuvieron esos sufijos).

\section{Conclusión}

Al analizar cada una de las lenguas descritas se han expuesto ya los comentarios pertinentes sobre sus sistemas morfológicos. Por tal motivo, a modo de cierre, se añadirán tan solo unas reflexiones muy breves.

En primer lugar, las lenguas auxiliares internacionales, tanto las dos analizadas como la mayor parte de las muchas que se han diseñado a lo largo de la historia, nacen con la intención de servir como medio de comunicación sencillo, estructurado y eficaz para cualquier persona, de modo que se consiga con ellas una comunicación neutra y válida para todos los seres humanos.

Partiendo de ahí, ese objetivo se intenta alcanzar, por lo que respecta al ámbito de la morfología, construyendo sistemas en los que se buscan la regularidad y la sistematicidad (con la supresión de la alomorfia y de los fenómenos morfonológicos), la precisión (con el establecimiento de categorías regulares) y la eliminación de las «deficiencias» semánticas que afectan a las lenguas naturales (vaguedad, polisemia y sinonimia).

En todo caso, como creemos haber mostrado al analizar, más superficialmente de lo que hubiéramos deseado, ambas lenguas, ese objetivo se convierte más en un deseo que en una realidad, aunque ello se manifiesta en diverso grado en cada lengua auxiliar concreta, como refleja la comparación establecida entre el volapük, lengua que tuvo un grande pero efímero éxito, y la langue bleue, que no llegó a pasar de simple proyecto.

\section{Bibliografía}

\section{Fuentes de las lenguas estudiadas}

\subsection{Sobre el volapük}

En línea: <www.volapük.com> [consulta: 15 de abril de 2018]. En esta web se alojan los siguientes documentos:

- Introduction: A Quick Look at Volapük. Archivo en formato pdf que presenta un resumen general de las características de esta lengua.

- Quick Volapük. Conjunto de diez lecciones que conforman un curso de nivel básico de esta lengua.

- Volapük Grammatical Forms. Gramática básica del volapük. 
- Bishop, B.R. (2012): Gramat Smalik Volapüka / Malgranda Gramatica de Volapuko. Gramática completa del volapük escrita en esperanto.

- Midgley, R. (2010): Volapük-English / English-Volapük Dictionary.

\subsection{Sobre la langue bleue}

Bollack, L. (1900): Grammaire abrégée de la langue bleue: bolak, langue internationale practique. Paris, Lèon Bollack (en línea: <http://gallica.bnf.fr/ark:/12148/ bpt6k202965p/f73.image> [consulta: 15 de abril de 2018]).

\section{Referencias bibliográficas}

Boolj, G. (2007): The Grammar of Words. An Introduction to Morphology. 2. ${ }^{\mathrm{a}}$ ed. Oxford, OUP.

Bosque, I. (1982): «La morfología». En Abad, F. y García Berrio, A. (eds.): Introducción a la lingüistica. Madrid, Alhambra, págs. 115-153.

Calero Vaquera, M.L. (1999): Proyectos de lengua universal. La contribución española. Córdoba, Cajasur.

Couturat, L. y Leau, L. (2001 [1903]): Histoire de la langue universelle. Ed. facsímil. Hildesheim, Georg Olms.

Comrie, B. (1996): «Natural and Artificial International Languages: A Typologist's Assessment». Journal of Universal Language, 1, págs. 35-55.

Díaz Hormigo, M.T. (2003): Morfología. Cádiz, Universidad de Cádiz.

DodD, W.S. (1990): «El esperanto y las lenguas artificiales». Estudios humanísticos. Filología, 12, págs. 105-129.

Eco, U. (1994): La búsqueda de la lengua perfecta. Barcelona, Crítica.

Edwards, J. (2013): «A Language for all the World». Language Teaching, 46.3, págs. 365-381.

Fernández Pérez, M. (1993): Las categorías gramaticales (morfológicas) en español. Santiago de Compostela, Universidad de Santiago de Compostela.

Galán Rodríguez, C. (2009a): Mundos de palabra. Utopías lingüísticas en la ficción literaria. Badajoz, Diputación de Badajoz.

- (2009b): «La invención de lenguas en la ficción literaria». En Jiménez Ruiz, J.L. y Timofeeva, L. (eds.): Estudios de lingüistica: Investigaciones lingüísticas en el siglo XXI. Alicante, Universidad de Alicante, págs. 103-130.

- (2012): «Lenguas universales». En Zamorano Aguilar, A. (ed.): Reflexión lingüistica y lenguas en la España del siglo XIX: Marcos, panoramas y nuevas aportaciones. München, Lincolm, págs. 417-442.

González Calvo, J.M. (1988): Estudios de morfología española. Cáceres, Universidad de Extremadura.

Grande Alija, F.J. (2001): «El problema de la comunicación internacional: las lenguas artificiales». Estudios humanísticos. Filología, 23, págs. 29-52.

Kamman, W.F. (1942): «The Problem of a Universal Language». The Modern Language Journal, 26.3, págs. 177-182. 
Libert, A. (2003): Mixed artificial languages. Muenchen, Lincom.

Martínez Gavilán, M.D. (2016): «La contribución de Caramuel a la creación de lenguas artificiales: características universales, lenguas filosóficas y lenguas secretas». Revista de investigación lingüistica, 19.1, págs. 77-106.

Matthews, P.H. (1980): Morfología. Introducción a la teoría de la estructura de la palabra. Madrid, Paraninfo.

Monnerot-Dumaine, M. (1960): Prècis d’interlinguistique générale et spéciale. Paris, Librairie Maloine.

PeI, M.A. (1974): «Artificial Languages: International (Auxiliary)». En Sebeok, T.A. (ed.): Current Trends in Linguistics, XII. The Hague: Mouton, págs. 999-1017.

Petersen de Piñeros, G. (1988): «La clasificación tipológica de las lenguas». Forma y Función, 3, págs. 17-32.

Robins, R.H. (1995): Lingüistica general. 4. ${ }^{a}$ ed. Madrid, Gredos.

Simone, R. (1992): Diario lingüístico de una niña. Barcelona, Gedisa. 